10.2478/gb-2020-0015

sciendo

\title{
Die Hebamme und ihre Benennungen im Siebenbürgisch-Sächsischen
}

\author{
Sigrid HALDENWANG \\ Dr.; Forschungsinstitut für Geisteswissenschaften Hermannstadt/ \\ Sibiu; E-Mail: sigridhaldenwang@yahoo.de
}

\begin{abstract}
This article covers midwives as such and their designations in the Transylvanian-Saxon vernaculars in detail with emphasis on the early documentary evidence in the first half of the 16th century as well as from the 18th century. The lexemes correlate their respective categories of word formation and show descriptive series of synonyms depicting at the same time the composition of the Transylvanian-Saxon vocabulary. Comments on the etymology of the word formations as well as on the midwife's profession are also included. The terms are taken from the Transylvanian-Saxon Dictionary and the North Transylvanian Saxon Dictionary as well as the specialist literature on vernacular.
\end{abstract}

Keywords: designation, midwife, Transylvanian-Saxon vernacular, composition of vocabulary, word formation, series of synonyms.

\section{Einleitung}

Im Beitrag wird auf die Hebamme und ihre Benennungen in den siebenbürgisch-sächsischen ${ }^{1}$ Mundarten näher eingegangen,

${ }^{1} \mathrm{Zu}$ den siebenbürgisch-sächsischen Mundarten vgl. Haldenwang, Sigrid: Das Siebenbürgisch-Sächsische in der Sprachlandschaft Siebenbürgen. In: Kriegleder, Wynfrid et al. (Hgg.): Deutsche Sprache und Kultur-Presse - Literatur-Geschichte in Siebenbürgen. Bremen 2009, S. 11-23. 
die sich schon urkundlich in Belegen der ersten Hälfte des 16. Jahrhunderts und 18. Jahrhunderts bezeugen lassen. Die Lexeme werden dabei verschiedenen Wortbildungsarten zugeordnet. Es folgen Erläuterungen zum Wortbildungsvorgang, zur Etymologie der Wortbildungen sowie zum Beruf der Hebamme. Die Benennungen sind dem Siebenbürgisch-Sächsischen Wörterbuch (SSWB) ${ }^{2}$, dem Nordsiebenbürgisch-Sächsischen Wörterbuch (NSSWB) ${ }^{3}$ sowie der mundartlichen Fachliteratur

2 Siebenbürgisch-Sächsisches Wörterbuch. Bisher 10 Bde erschienen. Bd. 1 (A-C) bearb. v. Schullerus, Adolf, Bd. 2 (D-F) bearb. v. Schullerus, A., Hofstädter, Friedrich u. Keintzel, Georg. Berlin 1924, 1926 (in einzelnen Lieferungenschon ab 1908 erschienen); Bd. 5 [RSalarist: alte Zählung] bearb. v. Roth, Johann, Göckler, Gustav, Berlin 1929-1931. Weitergeführt von einem Wörterbuchteam: Bd. 3 (G), Bd. 4 (H-J), Bd. 5 [K: neue Zählung] Berlin/Bukarest 1971-1975; Bd. 6 (L) 1993, Bd. 7 (M) 1998, Bd. 8 (N-P) 2002, Bd. 9 (Q-R) 2006, Bd. 10 (S-Sche) 2014 Bukarest [wird fortgesetzt]. [SSWB]. Das Wörterbuch will den Allgemeinwortschatz der Siebenbürger Sachsen erfassen; mit eingeschlossen sind diemundartliche Volks- und Kunstdichtung (Redensarten, Sprichwörter, Rätsel und Kinderspiele), Fachbenennungen sächsischer Handwerke, Entlehnungen aus dem Rumänischen und Ungarischen, Flurnamen, Ortsnamen, Pflanzennamen sowie Taufnamen (besonders Heiligennamen), die zahlreiche lautliche Varianten aufweisen oder auch im Sinne von Gattungsnamen auftreten. Aufgenommen werden auch Belege aus der siebenbürgischen Urkundensprache (von der Mitte des 13. Jahrhunderts bis zur Mitte des 19. Jahrhunderts), um die Bedeutung des mundartlichen Einflusses auf die deutsche Schriftsprache in Siebenbürgen und Geschichte der deutschen Sprache zu verdeutlichen. Die Bearbeitung erfolgt nach festgelegten wissenschaftlichen Grundsätzen, die Gliederung der einzelnen Wortartikel wird nach lautlichen, grammatikalischen und semantischen Kriterien durchgeführt.

3 Nordsiebenbürgisch-Sächsisches Wörterbuch.Bd. 1 (A-C) v. Krauß, Friedrich, bearb. v. Richter, Gisela Köln 1986, Bd. 2 (D-F) Köln 1990; Bd. 3 (H-M) Köln 1993, Bd. 4 (N-Sch) Köln 1995, Bd. 5 (SeZ) Köln 2006, Bd. 2-4 bearb. v. Richter, Gisela aufgrund der nachgelassenen Sammlungen von Krauß, Fr.; Bd. 5 bearb. v. Richter, G. † u. 
entnommen. Die Lexeme werden von der Wortbildung ausgehend, wie folgt gruppiert:

\section{Determinativkomposita ${ }^{4}$, die indigene Lexeme sind}

- in urkdl. u. mal. Belegen bezeugt:

1. UK ${ }^{5}$ : Subst. Simplex + 2. UK: Subst. Simplex

„Ammfrau“ bzw. „Amtfrau“

- Aufgabenkreis/Dienstverhältnis

Die Ammfrau, mal.,,omfrä““[f.] (H), ,ąm(t)frā“(B u. nsbg. verbr. $)^{6}$, ist in der Bedeutung, Wehmutter, Hebamme' in

Feßler, Helga aufgrund der nachgelassenen Sammlungen von Krauß, Fr. [NSSWB].Das Wörterbuch bearbeitet nach denselben Prinzipien, doch eigenen Methoden eingehend die nordsiebenbürgischen Mundarten: den Wortschatz der Bewohner von 48 Ortschaften, meist Dörfern, in den Kleinräumen Nösnerland und Reener Ländchen. Für das Nösnerland ist Bistritz, auch Nösen genannt, die Stadt mit starker Ausstrahlung auf die umliegenden Orte, für das Reener Ländchen ist es Sächsisch Reen (Regen). Gemeinsamkeiten der süd- und nordsiebenbürgischen Mundarten sind in der Grammatik und im Wortschatz feststellbar. In diesem Wörterbuch wird die siebenbürgische Urkundensprache nicht berücksichtigt. Die im Beitrag angeführten siebenbürgisch-sächsischen Ortsgemeinden sind in der Grundkarte des SSWB verzeichnet, die ab dem 7. Band (M) mitgegeben wird.

${ }^{4} \mathrm{Vgl}$. dazu die Formativstruktur des Determinativkompositums in: Fleischer, Wolfgang/Barz, Irmhild: Wortbildung der deutschen Gegenwartssprache. Tübingen 1992, S. 95 ff.

5 Da die Determinativkomposition der Ausdruckserweiterung zuzuordnen ist, wird von den unmittelbaren Konstituenten [UK] ausgegangen, die nach Fleischer (1974: 50), „diejenigen Konstituenten [sind], aus denen jede Konstruktion unmittelbar gebildet ist, in die sie sich auf der nächstliegenden Ebene zerlegen lässt". Fleischer, Wolfgang: Wortbildung der deutschen Gegenwartssprache. Leipzig 1974.

${ }^{6} \mathrm{Zu}$ folgenden Lautvarianten siehe: NSSWB, Bd. 1 (A-C), Spalte 245: „,amfrāa (Reen), „omfrā““ (Lu),,ą̧amtsfrāo“ (Wm), ,ạnfrạa “ (Bud, Eid, Nd-Wall, Tschi, Wall, Wl), ,untfrā“‘, auch „umtfrā" (Boo), „umtfrā““ (Nd-Ei). 
urkundlichen Belegen ab der ersten Hälfte des 16. Jahrhunderts in verschiedener Schreibweise belegt: „dy A $\mathrm{m}$ fra w “ (Burzenländer Steuerverzeichnis; 1536 Bre) $)^{7}$; „Amt fry” (Burzenländer Steuerverzeichnis; $1541 \mathrm{Wbch})^{8}$; ,So haben mein herrn den knecht frey gesprochen, dieweil 2 a mtfraw en die magd besehen und nichts beschwengert gefunden". Paul Joseph aus Marienburgklagt im Namen seiner Schwester gegen den Sohn des Hans Colwen wegen der Nichteinhaltung eines Eheversprechens (Gerichtsprotokoll; 1562, Kr) ${ }^{9}$. Die beiden erste Belege bezeugen, dass die Ammfrau schon um diese Zeit zu den Steuernträgern einer der Gemeinden des Burzenlandes gehört, wobei der Steuerbetrag nicht angegeben wird. So muss sie in einem Angestelltenverhältnis zu ihrer Gemeinde gestanden haben. Der folgende Beleg deutet darauf hin, dass es um Geburtshelferinnen geht, da sie zur Feststellung einer Schwangerschaft gerufen werden. Dazu führt der Sprachforscher und Volkskundler Adolf Schullerus ${ }^{10}$ an, dass um 1900 die Ammfrau gegen Jahresgehalt und geringe Wöchnerinnentaxen von der Gemeinde gewählt wird. Ein nordsiebenbürgischer Mundartbeleg bestätigt diese Angabe: ,sai hēszt ąmtfrau, dän dōt asz Īr ąmt, sai bəkit jo/von der Gemeinde/gialt" (etwa in dem Sinn: ,sie heißt Amtfrau, das ist ihr Amt, sie erhält ja von der Gemeinde ihren Lohn'; um 1900 S-Gg). Im Notfall hat sie die Taufe zu vollziehen, bezeugt in der Kirchenordnung des Reformators Honterus: „wo aber geschickte menner abgehen, mögen gut vnterrichtete weiber oder a mptfrawen in solcher not die kind tauff reichen"11. Ihr

7 Rechnungen aus dem Archiv der Stadt Kronstadt. Bd. 3. Kronstadt 1896, S. 20 f.

8 Ebd., S. 135.

9 Derzsi, Julia (Hg.): Quellen zur Geschichte der Stadt Kronstadt Band $X$-Das Gerichtsbuch des Kronstädter Rates (1558-1580). Kronstadt 2016, S. 36.

${ }^{10}$ SSWB, Bd. 1 (A-C), S. 99.

${ }^{11}$ Honterus, Johannes: Ausgewählte Schriften. Hrsg. von Oskar Netoliczka. Hermannstadt 1898, S. 75. 
Ehrentag ist der Tauftag, wo sie zur Belustigung der Gäste allerlei Späße aufführt, mit denen sie die Einsammlung von Geldgeschenken einleitet. Bezüglich des Geldgeschenks, das die Paten der Ammfrau geben, heißt es z.B.: „dər omfrā gądj əntsōəszjī̄ən“" (,der Ammfrau Gotenzinss ${ }^{12}$ geben'; um 1900 Zen); dazu erläuternd:,,də gądjəntsōəisz bətsōld a jēdər, dīdən ąf dər käiməsz wēər dər ōmfrā" (,den Gotenzins bezahlt jeder, der an dem Kirchweihfest teilnimmt, der Ammfrau'; 1960 Zen). Auch in das Taufbecken wird zuweilen eine Gabe gelegt. „Bei der Taufe legt der Vater des Kindes der Ammfrau in das Taufbecken [...] 6 Pfennig, die Taufzeugen geben nach Belieben etwas oder nichts" (Nachbarschaftsordnung; 1777 Gr-Schenk). Beim ersten Kirchgang, dem Einleiten, begleitet die „omfräa“ die junge Mutter. Nach dem Kirchgang wird ihr ein festliches Abschlussessen geboten, da sie von nun an die Wöchnerin und ihr Kind nicht mehr zu verpflegen verpflichtet ist ${ }^{13}$, dazu die zugedachte Entlohnung (ein Viertel Weizen, Speck, ein Brot), ferner von der Gemeinde eine geringe Geldtaxe ${ }^{14}$. Ein Beleg aus Nordsiebenbürgen bezeugt andererseits: „tsən fār weoxn git əm dər ąm(t)fraə āszn, neomətäąxāszn, dət auszšink“" (etwa in dem Sinn: ,bis zu vier Wochen nach der Entbindung gibt man der Ammfrau ein Nachmittagessen, Ausschenken genannt"; um $1930 \mathrm{Bud})^{15}$. Es bleibt aber weiter ein Vorrecht der Ammfrau, die Wöchnerin und den Täufling zu besuchen, sich bewirten zu lassen sowie ein freundschaftliches Verhältnis zu Mutter und Kind aufrecht zu erhalten.

${ }^{12}$ „Gotenzins“ (Gote i.S.v. Pate + Zins). Vgl. dazu Gote [1]: SSWB, Bd. 3 (G), S. $276 f$.

${ }^{13}$ SSWB, Bd. 1 (A-C), S. 338; siehe „Ausschank“. Vgl. dazu Hillner, Johann: Volksthümlicher Glaube und Brauch bei Geburt und Taufe im Siebenbürger Sachsenlande. In: Programm des evang. Gymansiums in Schässburg und der damit verbundenen Lehranstalten (Schuljahr 1876/77), S. 46.

${ }^{14} \mathrm{Vgl}$. SSWB, ebd.

${ }^{15}$ NSSWB, Bd. 1 (A-C), Spalte 246. 
Adolf Schullerus ${ }^{16}$ verdeutlicht, dass der Ammfrauendienst lange Zeit vielfach neben den amtlich angestellten Ammfrauen, von älteren Frauen, die ohne ärztliche Ausbildung und behördliche Genehmigung Kranke behandelten, ausgeübt wurde. Diese Frauen waren berühmt durch ihre Heilmittel, aus bestimmten Pflanzen hergestellten Salben, die besonders gut „schmieren“ und „ziehen“, d.h. schmerzhafte Körperstellen oder Wunden heilen können. Daher wurden sie oft von weither aufgesucht, worauf folgender Beleg deutet: „,ggīt kēn N. ${ }^{17}$ bä dä fərmērt omfrā!"“ (,er geht nach N. zu der berühmten Ammfrau'; um 1900 Schbg) $)^{18}$; dann auch auf eine Hausfrau bezogen: „dā kạ̄n tsă̄n wä ən omfrǟ"(,die kann massieren wie eine Ammfrau'; o.O., ssbg.) ${ }^{19}$; auf eine Kurpfuscherin gemünzt: ,sai hālt si $\chi$ wāi an ąmtfrā" (etwa in dem Sinn ,sie, die Kurpfuscherin, hält sich für eine ausgebildete Ammfrau'; Mö) ${ }^{20}$; auch scherzend in einem Kartenspiel gesagt, wenn dem Partner hohe Points geworfen (d.h. geschmiert) werden: „dī šmīərt dər wâ̄ ən omfrǟ“ (wörtlich: ,die schmiert dir, wie eine Ammfrau'; o.O., ssbg.). ${ }^{21}$. Wenn eine Sache schwer vorwärtsgeht, z.B. ein Spieler beim Kartenspiel nicht rasch eine Karte zuwirft, gilt die Redensart: „selə mər nō dər omfrä šäkən?“(,sollen wir nach der Ammfrau schicken?'; gemeint ist: ,soll die Ammfrau dich massieren, um dir auf die Sprünge zu helfen?‘; Med) ${ }^{22}$.

- Die Ammfrau als Kinderbringerin:

Den Kindern wird erzählt, dass die Ammfrau die Kinder bringt, deshalb gilt sie in der Kinderwelt als Bringerin der jüngeren Geschwister. Der Volskundler Johann Hillner $^{23}$ bemerkt

\footnotetext{
${ }^{16} \mathrm{SSWB}$, ebd.

${ }^{17}$ Fiktive Ortschaft.

${ }^{18}$ SSWB. Bd. 1 (A-C), S. 99.

${ }^{19}$ Ebd.

${ }^{20}$ NSSWB, ebd.

${ }^{21}$ SSWB, ebd.

${ }^{22}$ Ebd.

${ }^{23}$ Hillner, S. 17.
} 
diesbezüglich:,,die /omfrā/ bringt den neugeborenen Sprössling aus ihrer Tasche, oder aus einer alten Darre (mal. dar,Vorrichtung zum Obstdörren'/herbei“; Schbg). Folgende Belege sind Antworten auf die Frage, woher die Kinder kommen: die Ammfrau kann selbst darauf antworten: ,e $\chi$ hun əd äm gəšpren gəfäšt" (,ich habe es aus der Quelle eines Flusses gefischt'; R-au) ${ }^{24}$; doch sind es meist Antworten anderer Erwachsenen: „də omfrä

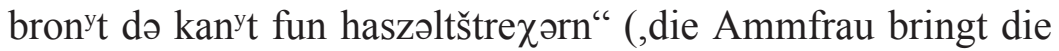
Kinder von den Haselnusssträuchern“; um 1900 Msch); „də ąmtfrā holt də klī kandər əusz dəm nąszbōm, əusz dər wəit“" (,die Ammfrau holt die kleinen Kinder aus dem Nussbaum, von Weide“; Wl ${ }^{25}$; , də kä̀nt kun än dər bạ̄y ərōf gəflōszən, dərniu šēpt də omfrā sə än də kōp“(,die Kinder kommen in dem Bach herab geflossen, danach werden sie von der Ammfrau mit einem Krug aus dem Bach geschöpft; Katz) ${ }^{26}$; auch „ət šwūm alts ə kradər än dər bauox, dərniä hūld ət də omtfrā" (etwa in dem Sinn: ,das Kind schwamm in der Gestalt eines Frosches in dem Bach, dann holte es die Ammfrau heraus '; um 1960 Tra ${ }^{27}$; dazu nicht lokalisiert: ,də omfrā hod /das Kind/äm papeiər ägəwäkəlt brōxt"(,die Ammfrau hat das Kind im Papier eingewickelt gebracht'; o.O. ssbg. ${ }^{28}$. Dazu auch ein Sprichwort, das inhaltlich der deutschen Schriftsprache entspricht: „ba filn ąm(t)frānə get dət kant tsəgront"(,bei vielen Ammfrauen geht das Kind zugrunde '; entspricht: ,viele Köche verderben den Brei'; Zep) ${ }^{29}$.

- etymologisch betrachtet: 1 . UK zu Amme gestellt; den nsbg. Wortformen mit -t- Einschub liegt volksetymologische Anlehnung an ,Amt' zugrunde $^{30}$.

${ }^{24}$ Ebd.

${ }^{25}$ NSSWB, Bd. 1 (A-C), Spalte 246.

${ }^{26}$ Siehe Beleg unter „Bach“ (SSWB, Bd. 1[A-C], S. 367).

${ }^{27}$ SSWB, ebd.

${ }^{28}$ Ebd., S. 99.

${ }^{29}$ NSSWB, ebd.

${ }^{30}$ Siehe Ommfræ (SSWB, Bd. 1 [A-C], S. 99) und „Ammfrau“ (NSSWB, Bd. 1 [A-C], Spalte 245f.). 
- Wortbildungsvorgang: 1. UK Amme, mal. om (ssbg.), om(t)(nsbg.) +Frau, mal. frā (ssbg.) + frā (nsbg.) > Ammfrau, mal. omfrä (ssbg.) bzw. mal. om(t)frā (nsbg.).

1. UK: Vb.stamm + 2. UK: Subst. Simplex

„Hebamme“: Die Hebamme, mal. „hēbam“ [f.] (H u. B), ist heute eine in einer besonderen Lehranstalt ausgebildete, staatlich geprüfte Geburtshelferin. Das Lexem ist in siebenbürgischen urkundlichen Quellen in unterschiedlicher Schreibweise seit der 2. Hälfte des 18. Jahrhunderts bezeugt, z.B.: „,so sey Capp zur H e w a $m$ m e gegangen“ $(1760)^{31}$; „Anna Schneiderin der örmenyescher Hebammin Tochter" (1762) $)^{32}$ und „H e w a n n e" (18. Jh.). ${ }^{33}$. Das der deutschen Schriftsprache angehörige Lexem ist im Siebenbürgisch-Sächsischen wenig belegt: z.B. „heəwan“ (um 1900 Gr-Schenk); „hēwan“ (1930 Gr-A). Dass es sich um eine staatlich Angestellte handelt, bezeugt der Beleg: „də hēbam dīt dən dạ̄intst ä Rōž äm diszpänszār" (,die Hebamme versieht ihren Dienst in der Gemeinde Rosch, im Ambulatorium'; 1960 Mard); dann auch scherzhaft in einer Redensart auf einen guten Redner bezogen: „disəm huat də hēbam dət maul gāt ậfgəräszn" (wörtlich: ,diesem hat die Hebamme den Mund gut aufgerissen“; 1935 B).

- etymolgisch betrachtet: umgedeutet aus ahd. hevianna ,hebende Ahnin': zu ahd. hevan (heben) + ana ,Ahne, Großmutter'; angelehnt an Amme; ,heben' bezieht sich offenbar auf das Hochheben des Kindes unmittelbar nach der Geburt ${ }^{34}$.

${ }^{31}$ Nationalarchiv Hermannstadt (früher Archiv der Stadt Hermannstadt u. der Nationsuniversität).

${ }^{32}$ Ebd.

${ }^{33}$ Siebenbürgische Vierteljahresschrift, 1931, S. 196.

${ }^{34}$ Vgl. Wahrig, Gerhard et al. (Hgg.): Brockhaus Wahrig - Deutsches Wörterbuch, Bd. 3 (G-JZ). Stuttgart 1981, S. 442 und Kluge Etymologisches Wörterbuch der deutschen Sprache. Bearb. v. Elmar Seebold. Berlin ${ }^{23}$ 1995, S. 361. 
- Wortbildungsvorgang: 1. UK heb, mal. hēb, heəw, hēw (Vb. stamm von heben, mal. hēbn, heəwən, hēwən) +2 . UK Amme, mal. am bzw. an [Ahne entsprechend] > Hebamme, mal. hēbam, heəwan, hēwan.

- nur in mal. Belegen bezeugt:

1. UK: Subst. Simplex + 2. UK: Subst. Simplex

„Bienmutter": Die eigentliche Bedeutung der Wortbildung Bienmutter, mal. ,bą̄əmotər"635[f.] (ssbg.) und ,bąāimątər ${ }^{6636}$ (nsbg.), ist ,Bienenkönigin“. Im übertragenen Sinn kann nur nordsiebenbürgisch die Bedeutung, Hebamme ${ }^{37}$ belegt werden. So heißt es: ,gik nō dərbạ̄imątər!“" (,geh hole die Bienmutter'; gesagt, wenn die Geburt naht; o.O., nsbg.).

- Wortbildungsvorgang: 1. UK Biene, mal. bą̄ə, bą̄i + 2 . UK Mutter, mal. motər, mątər > Bienmutter, mal. bą̄əmotər, bąimątər.

\section{Simplex, ein indigenes Subst.}

„Große“: Die Grundbedeutung des Wortes Große, mal. „grīsz" [f.] (ssbg. auch andere Lautformen, ,grūsz", nsbg., auch andere Lautformen), ist eigentlich ,Großmutter". Die Bedeutung ,Hebamme“ ist nur in einigen nordsiebenbürgischen Gemeinden belegt. Den Kindern wird erzählt: „dāi gruisza holt də kändər ausz dəm wạ̄lt" (,diese Große holt die Kinder aus dem Wald“; Schbk, auch Tschi).

- etymologisch betrachtet: siehe dazu Adj. gross (3a) subst., mal. grōs und grōsjə in verschiedenen Lautvarianten in

${ }^{35}$ Vgl. SSWB, Bd. 1 (A-C), S. 693.

${ }^{36}$ Vgl. NSSWB, ebd., Spalte $1094 f$.

${ }^{37}$ Keintzel, Georg: Nösner Idiotismen. In: Festgabe der Stadt Bistritz. Bistritz 1897, S. 48 und Kisch, Gustav: Nösner Wörter und Wendungen. Ein Beitrag zum siebenbürgisch-sächsischen Wörterbuch. Bistritz 1900, S. 101. 
Ortschaften des Moselfränkischen und Ripuarischen verbreitet in der Bedeutung, Großmutter ${ }^{638}$.

\subsection{Simplex, als 2. $U K$ in Determinativkomposita}

1. UK: Subst. Simplex + 2. UK: Subst. Simplex

„Buschgroße“: Die Buschgroße, mal. „bäšgrīsz“ [f.], ist eine scherzhafte Benennung der Hebamme, die in einer Schürze die Kinder aus dem Wald bringt (Umgebung von $\mathrm{H}$ ) ${ }^{39}$, und bäšgrūszi (nsbg. $)^{40}$ :

- Wortbildungsvorgang: 1. UK Busch, mal. bäš (i.S.v. Wald)

+2. UK Große, mal. grīsz bzw. grūszi.

1. UK: Determinativkompositum + 2. UK: Subst. Simplex

„Lindbuschgroße“: Von der Lindbuschgroße, mal. „lampəšgruiszo“ [f.] (um $1930 \mathrm{~S}-\mathrm{Gg}$, auch Lechn, Wm), wird den Kindern erzählt, dass sie die Neugeborenen bringt. Darauf deutet der Beleg: „dəlampəšgruiszəhuat dət jąyəltxi ausz dəm Lampəš gəgəholt" (,die Lindbuschgroße hat das Jüngelchen aus dem Lindbusch ${ }^{41}$ geholt $;$; $\left.-\mathrm{Gg}\right)^{42}$.

- Wortbildungsvorgang: 1. UK Lindbusch, mal. Lämpəš (nsbg. Fl.N.) + Große, mal. gruiszə > Lindbuschgroße > mal. lampəšgruiszə.

1. UK: Vb.stamm + 2. UK: Subst. Simplex

„Schmiergroße“: Die Benennung Schmiergroße, mal. „šmiorgrīsz" [f.], geht darauf zurück, dass die Hebammen früher auch schmierten, d.h. durch Einschmieren mit Salben, die sie selbst herstellten, Heilversuche unternahmen; sie betrieben auch Kurpfuscherei: in diesem Sinn der Beleg: „tsə desər ậldə

${ }^{38}$ Rheinisches Wörterbuch, bearb. u. hrsg. von Josef Müller. Bd. 2 (EG). Berlin 1931, Spalte 1437.

${ }^{39}$ SSWB, ebd., S. 836.

${ }^{40}$ Keintzel, ebd.

${ }^{41}$ Nsbg. häufig belegter Flurname.

${ }^{42}$ Vgl. NSSWB, Bd. 3 (H-M), Spalte 1316. 


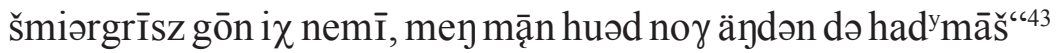
[etwa in dem Sinn: ,zu dieser alten Kurpfuscherin gehe ich nicht mehr, mein Mann leidet immer noch an der Schlafkrankheit' (um $1930 \mathrm{H}$, auch Baa)].

- Wortbildungsvorgang: 1. UK schmier: Vb.stamm (zum Vb. schmieren, mal. šmiərən), mal. šmiər +2 . UK Große, mal. grīsz $>$ Schmiergroße $>$ mal. šmiorgrīsz.

\subsection{Simplex als 2. $U K$ in einer Hybridbildung ${ }^{44}$}

1. UK: exogenes Subst. +2. UK: indigenes Subst.

„Baləgroße“: Die Grundbedeutung der Wortbildung Baləgroße, mal. „baləgrīsz"“ [f.] (ssbg.) und „bąləgrīsz" (nsbg.), ist zunächst ,Großmutter", ,Urahne' mit der negativen Konnotation des Verächtlichen, Schimpflichen. Das Wort ist um 1900 allgemein meist in der Verstärkung „wält baləgrīsz" (,wilde Großmutter') aber auch ohne dem Beiwort, wild“ (das aber mitverstanden wird) in Wendungen verbreitet, die Unwillen, Zorn zum Ausdruck bringen, wie: „gą̧̣k än dẹ wält baləgrīsz!““ (wörtlich: ,gehe in deine wilde Großmutter'!; um 1900 Schbg). Davon ausgehend, hat sich die Bedeutung ,Hebamme“ entwickelt: ,gậnk tsə dejər baləgrīsz!“ (,geh zu deiner Hebamme“;

${ }^{43} H_{a d}{ }^{y} m \bar{a} s ̌<$ ung. ha g y má s ,Typhus, Schlafkrankheit‘.

${ }^{44}$ Unter Hybridwortbildung bzw. Mischwortbildung wird der Prozess der Entstehung der Hybrid- bzw. Mischbildungen verstanden. Die Hybridbildung wird dem Determinativkompositum zugeordnet. Die Hybridbildung/Mischbildung ist eine komplexe Komposition, eine Kombination aus indigenen (heimischen, muttersprachlichen) und exogenen (fremdsprachlichen) Einheiten. In unserm Fall können die exogenen Elemente als Erstglieder mit einem indigenen Lexem verbunden werden und umgekehrt. Vgl. dazu Dargiewicz, Anna: Fremde Elemente in Wortbildungen des Deutschen. Zu Hybridbildungen in der deutschen Gegenwartssprache am Beispiel einer raumgebundenen Untersuchung in der Universitäts- und Hansestadt Greifswald. In: Grabarek, Józef (Hg.): Schriften zur diachronen und synchronen Linguistik. Bd. 10. Frankfurt am Main 2013, S. 93. 
Kl-Schenk). Auch heißt es im vertraulichen Spott Kindern gegenüber: „sä $\chi$, ded äsz den baləgruisz“ (,sieh, das ist deine Hebamme'; um 1970 Gr-Schenk).

- Wortbildungsvorgang: 1. UK Balə + 2. UK Große, mal. grīsz bzw. gruisz $>$ mal. baləgrīsz bzw. bąləgrīsz.

- Eine befriedigende etymologische Erklärung dieser Wortbildung ${ }^{45}$ kann nicht gegeben werden. Adolf Schullerus verweist auf rumänisch b a $\breve{a}^{46}$ besonders in der Verstärkung ba lă spurcată (,wüstes, schmutziges Weib') im Rumänischen Südsiebenbürgens als Schimpfname vielfach gebraucht: tu b( o) a lă spurcată (,du schmutziges Weib'). Die zugrundeliegende Bedeutung ist jedoch die, von Geifer beschmutzte alte Frau“. Der Wortbildung „Baləgrīsz“" soll ursprünglich diese Bedeutung zugedacht worden sein, davon ausgehend der Bedeutungswandel zur, wilden, gespenstischen Großmutter' und auch übertragen auf die ,Hebamme‘.

\section{Simplex ein exogenes Subst., ung. Herkunft}

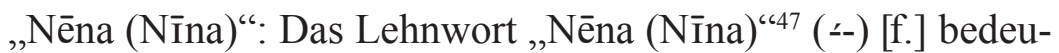
tet eigentlich ,Tante' im engeren und weiteren Sinn und gilt besonders als, Anrede für eine ältere Fau, ältere Verwandte auf dem Land'. Dazu ist auch die Bedeutung, Hebamme' bezeugt. So heißt es als Antwort auf die Frage, woher die Kinder kommen, z.B.: ,ən noina hoed ət broe $\chi \mathrm{t}^{\text {" }}$ (,eine Hebamme hat es gebracht"; Kl-Schenk) ${ }^{48}$; auch: „də nēna holt də kändər" (,die Hebamme holt, bringt die Kinder'; Win) ${ }^{49}$.

${ }^{45}$ Vgl. dazu SSWB, Bd. 1 (A-C),S. 397f.

${ }^{46}$ Siehe bală ${ }^{1}$ [f.] ,Untier'. (Tiktin, Hariton: Rumänisch-Deutsches Wörterbuch. Bd. 1 (A-C). Wiesbaden ${ }^{2}$ 1968, S. 271.

${ }^{47}$ In verschiedenen Vokalvarianten belegt, siehe: SSWB, Bd. 8 (N-P), S. 89 und NSSWB, Bd. 4 (N-Sch), Spalte 83.

${ }^{48}$ Korrerspondenzblatt des Vereins für siebenbürgische Landeskunde, 1902, S. 23.

${ }^{49}$ NSSWB, Bd.4 (N-Sch), ebd. 
- Herkunft < ung. néni, Tante, ältere Verwandte, ältere Schwester, alte Frau; auch als Anrede, an eine (ältere) Frau ${ }^{650}$; mit s.s. Bedeutungswandel.

„Szila“: Dem Lehnwort „Szila“ $\left(s^{-}\right)^{51}$ [f.] liegt auch die Grundbedeutung, Großmutter, Urgroßmutter ${ }^{6}$ zugrunde, doch bezeugen zwei Belege auch die Bedeutung ,Hebamme': „dąt wärr dəm kant də szila“(etwa in dem Sinn: ,das wäre die Hebamme des Kindes'; um 1930 W1). Dazu in einer anschaulichen Redensart: ,dəwiszt ku, wun män szila an brəut asz, fum kirfi $\chi$ kit, mā'm bōrtn“ (wörtlich: ,du wirst kommen, wenn meine Hebamme eine Braut ist, und mit dem Borten vom Friedhof kommt'; das heißt ,am Nimmerleinstag'; o.J. Wl).

- Herkunft: Das Wort ist nach Kisch ${ }^{52}<$ ung. mal. s z il a n y ó ,Großmutter ${ }^{6}$ entlehnt, der ungarische Lexikograf Halász ${ }^{53}$ führt ung. s z ü le an ,glbed.'; mit s.s. Bedeutungswandel.

Simplex als 2. UK in Hybridbildungen

1. UK: Vb.stamm (Vb. schmieren [indigen]) + 2. UK: exogenes Subst. Simplex

„Schmiernēna“: Die Benennung „Schmiernēna“, mal. „šmiarnēna“ [f.], ist darauf zurückzuführen, dass die Hebammen sich auch als Kurpfuscherinnen betätigten; s. dazu Schmiergroße (um 1900 nsbg.).

- Wortbildungsvorgang: 1. UK schmier (Vb.stamm zu schmieren), mal. šmiar+ 2. UKnēna $>$ Schmiernēna $>$ mal. šmiarnēna.

1. UK: indigenes Subst. + 2. UK: exogenes Subst.

${ }^{50}$ Benkő, Loránd et al. (Hgg.): A magyar nyelv tőrténeti-etimológiai szótára (Historisch-etymologisches Wörterbuch der ungarischen Sprache). Bd. 2 (H-Ó), Budapest 1970, S. 1013.

${ }^{51}$ NSSWB, Bd. 5 (Se-Z), Spalte 235.

${ }^{52}$ Kisch, ebd., S. 133.

${ }^{53}$ Halász, Előd: Magyar-Német Szótár (Ungarisch-Deutsches Wörterbuch). Bd. 2, Budapest ${ }^{11} 1994$, S. 891. 
„Buschszila“: Die Wortbildung „Buschszila“, mal. „,bäšszila“" [f.], geht darauf zurück, dass man den Kindern erzählte,die Hebamme bringe die Kinder aus dem Wald ${ }^{54}$.

- Wortbildungsvorgang: 1. UK Busch, mal. bäš +2 . UK szila $>$ Buschszila > mal. bäšszila.

„Grabszila“: Die Benennung „Grabszila“, mal. „gruəfszila“ [f.], geht darauf zurück, dass man den Kindern erzählte, die Neugeborenen grabe die Hebamme aus der Erde aus. Dazu der Beleg: „,əgruəfszila griaft də kandər äusz dər iart“ (,sie gräbt die Kinder aus der Erde aus'; Zep $)^{55}$.

- Wortbildungsvorgang: 1. UK Grab, mal. gruəf + 2. UK szila $>$ Grabszila $>$ mal. gruəfszila.

\section{Simplex, ein exogenes Subst., rum. Herkunft}

„Moašə“: Das Lehnwort „Moašə“ [f.] in der Bedeutung „Hebamme' ist nur nsbg. (Eid, Pas, Tschi) ${ }^{56}$ belegt. Den Kindern erzählte man, „də moašə“ bringe die Neugeborenen aus einem Birnen- oder Pflaumenbaum (um 1930 Pas). Ein aussagekräftiger Beleg, der darauf deutet, dass diese Benennung allgemein im Gebrauch war, doch ohne genaue Zeitangabe, lautet: „dəa ąln hu mạ̄ešə gəsōt, dật asz mi wae blāšs, həit sō mər mēszt ąmtfrau“ (etwa in dem Sinn: ,die ältere Generation hat eher das Lehnwort für die Bezeichnung der Hebamme gebraucht, das ist mehr wie rumänisch, heute sagen wir meist Amtfrau'; o.J. Wl).

- Herkunft: < rum. m o a ș ă ,glbed.' in die Ma. eingelautet.

${ }^{54}$ Kisch, ebd., S. 16.

${ }^{55}$ Vgl. NSSWB, Bd. 2 (D-G), Spalte 1323.

${ }^{56} \mathrm{Zu}$ andern Vokalvarianten vgl. NSSWB, Bd. 3 (H-M), Spalte 1577. 


\section{Indigenes explizites Suffixderivat ${ }^{57}$}

„Batschlerin“: Die Batschlerin, mal. „batšlərän“ [f.], ist eine Spottbezeichnung der Hebamme, die früher gerne auch Kurpfuscherei betrieben hat (um 1900 Agn).

- Dazu der Wortbildungsvorgang: Batschler i.S.v. Pfuscher ist ein Suffixderivatmit deverbaler $\mathrm{DB}<\mathrm{Vb}$. batscheln [DB batsch + Suff. -eln] $>$ Suff.abfall des Vb. + subtantivbildendes Suff.-ler [DB batsch + Suff. -ler] $>$ Batschler + substantivbildendes Suff. -in $>$ Batschlerin $>$ mal. batšlərän.

- etymologische Angaben: Das Vb. ,batscheln' ist zu bairisch herum bázzn, in etwas Schmierigem herumlangen, herumgreifen'sowie bázln, ,medizinieren, besonders Hausmittel brauchen', zu stellen ${ }^{58}$.

\section{Schlussfolgerungen}

Ausgehend von der Wortbildung wurden zunächst Determinativkomposita angeführt, die indigene Lexeme sind. Dazu gehört die „Ammfrau/Amtfrau“, mal. „omfrǟ“[f.] (H), ,ąm(t)frāa“ (B) sowie die „Hebamme“, mal. „hēbam“[f.](H u. B). Dass der Hebammenberuf schon in seiner ersten Form seit der ersten Hälfte des 16. Jahrhunderts bezeugt ist, bestätigen zwei urkundliche Belege, die darauf deuten, dass die „Ammfrau/Amtfrau“ um diese Zeit in einem bestimmten Arbeitsverhältnis zu ihrer Gemeinde stand. Die Hebamme selbst, die der deutschen Standardsprache angehört, ist hauptsächlich ab dem 18. Jahrhundert in verschiedener Schreibweise bezeugt, ein nordsiebenbürgischer Mundartbeleg verdeutlicht aber, dass sie heute eine in einer besondern Lehranstalt ausgebildete, staatlich geprüfte

${ }^{57}$ Siehe dazu Suffigierung (Ergebnis Suffixderivat) unter „Explizite Derivation" bei Fleischer/Barz (1992: 46).

${ }^{58}$ Schmeller, J(ohann) Andreas: Bayerisches Wörterbuch. Bd. 1 bearb. v. Karl Fromann. München 1872, S. 314. 
Geburtshelferin ist. Zu dieser Wortbildungsart gehört auch die „Bienmutter", mal. „bạ̄əmotər"[f.] (ssbg.), und „bạaimątər" (nsbg.), die im übertragenen Sinn auch ,Hebamme“ bedeutet. Im Rahmen der indigenen Simplizia wurde zunächst auf „die Große“, mal. ,gruisza“[f.], auch ,gruisz" (nsbg.), deren Grundbedeutung, Großmutter, Urahne' ist, dann aber auch im übertragenen Sinn ,Hebamme‘ bedeutet, näher eingegangen. Mit dem Simplex sind folgende Determinativkomposita gebildet worden: „Buschgroße“, mal. „bäšgrīsz“[f.](ssbg.), „,bäšgrūszi“" (nsbg.), „Lindbuschgroße“, mal. „lampəšgruisza“[f.](nur nsbg.), „Schmiergroße“, mal. „šmiərgrīsz"[f.] (nur ssbg.), eine Benennung, die darauf zurückgeht, dass früher auch Frauen, die nicht für diesen Beruf ausgebildet waren, durch Einschmieren mit Salben, die sie selbst herstellten, Heilversuche unternahmen und Kurpfuscherei betrieben. Dazu gehört auch die Hybridbildung „Baləgroße“", mal. „,baləgrī̄sz“ [f.](ssbg.) und „,bąləgrīsz"“ (nsbg.), zunächst in der Bedeutung, Großmutter, Urahne " und im übertragenen Sinn auch ,Hebamme . Im Rahmen der exogenen Simpliza sind die aus dem Ungarischen entlehnten Lexeme „Nēna“ bzw. „Nīna“ [f.] (ssbg. und nsbg.) und „Szila“ [f.] bezeugt, die im Bezug auf das ungarische Ausgangswort einen siebenbürgisch-sächsischen Bedeutungswandel aufweisen. Mit diesen Lehnwörtern sind die Hybridbildungen „Schmiernēna“, mal. „šmīərnēna“ [f.] (nur nsbg.), „Buschszila“, mal. „bäšszila“ [f.] (nur nsbg.) und „Grabszila“, mal. „gruəfszila“ [f.], (nur nsbg.), entstanden. Nur als Simplex belegt, ist das aus dem Rumänischen entlehnte Lexem „Moašə“ [f.] (nur nsbg.). Der angeführte Mundartbeleg verdeutlicht, dass dieses Lehnwort schon sehr früh, doch ohne Zeitangabe bei der älteren Generation im Gebrauch war, dann aber von der „Amtfrau“ ersetzt wurde. Letztlich konnte auch ein indigenes explizites Suffixderivat aus einer Ortschaft belegt werden, die „Batschlerin“, mal. „batšlərän“" [f.](nur ssbg.), als Spottbezeichnung der Hebamme, die auch Kurpfuscherei betrieben hat. 
Bei einigen Benennungen (siehe: Ammfrau, Hebamme; Batschlerin und Große) wurde auf deren Etymologie aus historischer Sicht näher eingegangen, bei den exogenen Simplizia auf die Herkunft derselben und bei den Determiantivkomposita bzw. den Hybridbildungen ist der Wortbildungsvorgang verdeutlicht worden.

Die Mundartbelege zu den Benennungen umfassen lückenhaft den Zeitraum um „1900 bis etwa um 1970“. Einige Benennungen sind nur süd- oder nur nordsiebenbürgisch bezeugt. Das ist einerseits auf jahrhundertelange sprachliche Mischung und Ausgleich im ganzen Mundartgebiet zurückzuführen, andererseits wurde bei Mundarterhebungen wohl nicht immer gezielt nach den mundartlichen Benennungen der Hebamme gefragt. Auch hat die etappenweise Auswanderung der Siebenbürger SachsengroßeLückenindersiebenbürgisch-sächsischenSprachlandschaft verursacht. Was das nordsiebenbürgischeanschauliche Lehnwortgut betrifft, könnte auch der nachbarliche Einfluss eine bedeutende Rolle gespielt haben.

Die Mundartbelege lassen erkennen, dass in der Kinderwelt die Hebamme in ihren verschiedenen Benennungen als Kinderbringerin gilt. Sie bringt, holt die Kinder von, aus einem bestimmten Ort. Die Mundartbelege sind Antworten mit vielfältigen lokalen Angaben auf die Frage, woher die Kinder kommen.

Die Benennungen ergeben eine stattliche Synonymenreihe, die auch die Beschaffenheit des siebenbürgisch-sächsischen Wortschatzes veranschaulichen: indigenes (heimisches muttersprachliches) Wortgut zu dem auch ein Lexem gehört, das auf das Moselfränkische und Ripuarische hinweist (siehe Große) sowie ein Lexem, dessen Ausgangsbasis dem Bairischen zugeordnet wird (siehe Batschlerin). Dazu kommt exogenes Wortgut, aus dem Ungarischen entlehnte Simplizia (siehe dort) mit siebenbürgisch-sächsischem Bedeutungswandel und den damit belegten Hybridbildungen sowie das angeführte rumänische Lehnwort (siehe dort). 


\section{Schreibkonventionen ${ }^{59}$}

Abkürzungen

f. $=$ feminin

$\mathrm{DB}=$ Derivationsbasis

F1.N = Flurname

glbed. = gleichbedeutend

i.S.v. = im Sinne von

Ma. = Mundart

mal. = mundartlich

nsbg. $=$ nordsiebenbürgisch

o.J. = ohne Jahr

o.O. = ohne Ort

rum. = rumänisch

s.s. = siebenbürgisch-sächsisch

ssbg. = südsiebenbürgisch

Subst. $=$ Substantiv

subst. $=$ substantiviert

Suff. $=$ Suffix

ung. = ungarisch

urkdl. = urkundlich

$\mathrm{Vb} .=$ Verb

verbr. $=$ verbreitet

Zur Lautschrift

Die Vokale

Die Lautung der Vokale entspricht im Allgemeinen der hochsprachlichen, kleine Unterschiede werden in der Schreibung nicht berücksichtigt.

Abweichend von der Schriftsprache sind zu lesen:

$a i=\mathrm{a}-\mathrm{i}$

$\ddot{a} u=a ̈-u$

$e i=\mathrm{e}-\mathrm{i}$

$i e=\mathrm{i}-\mathrm{e}$

${ }^{59}$ Die Mundartwörter werden in Belegen klein geschrieben. 
$o e=\mathrm{o}-\mathrm{e}$
$u e=\mathrm{u}-\mathrm{e}$

Besondere Lautzeichen:

$\mathrm{a}=$ dumpfes a

$\hat{\mathbf{1}}=$ geschlossener Hintergaumenlaut ohne Lippenrundung (Reduktionsvokal zwischen i und ü, wie rumänischî)

$\partial=$ Murmel-e (auch in betonten Silben).

Kürze wird nicht bezeichnet, Länge durch darüber gesetzten geraden Strich $\bar{a}$

Die Konsonanten

$p, t, k \quad$ meist nicht behauchte Fortes

$\chi \quad$ stimmloser Ich-Laut

$x \quad$ stimmloser Ach-Laut

$s z \quad$ stimmloses $\mathrm{s}$

s $\quad$ stimmloses sch

ts $\quad$ stimmloses $\mathrm{z}$

$t \check{s} \quad$ stimmloses tsch

y Gutturalnasal ng

$b, d, g \quad$ stimmhafte Lenes

$j \quad$ stimmhafter Ich-Laut

$\gamma \quad$ stimmhafter Ach-Laut

$s \quad$ stimmaftes s

Ž stimmhaftes sch

$d s \quad$ stimmhaftes $\mathrm{Z}$

$d \check{z} \quad$ stimmhaftes tsch

${ }^{y}$ vor oder nach den Konsonanten d, t, 1, n zeigt Moullierung an.

Auslautendes Endungs-n fällt im Südsiebenbürgischen vor nachfolgendem Konsonanten, außer vor d, t, z, n und h, meist aus (Eifler Regel). 


\section{Ortssigel}

Abkürzung

deutsch / rumänisch Kreis/judeţ: deutsch /

rumänisch

Agn

$=$ Agnetheln/Agnita

$=$ Hermannstadt $/$ Sibiu

B

$=$ Bistritz/Bistriţa

= Bistritz/Nassod/Bistriţa/

Năsăud

Baa

$=$ Baaßen $/$ Bazna

$=$ Hermannstadt/Sibiu

Boo

$=$ Bootsch $/$ Batoș

= Mureș

Bre

$=$ Brenndorf $/ \mathrm{Bod}$

$=$ Kronstadt $/$ Brașov

Bud

= Budak/Budacu de Jos = Bistritz/Nassod/Bistriţa/ Năsăud

Eid

$=$ Eidau/Viile Tecii

= Bistritz/Nassod/Bistriţa/ Năsăud

Gr-A

$=$ Großalisch/Seleuşu

= Mureș

Gr-Schenk

$=$ Großschenk/Cincu

$=$ Kronstadt $/$ Brașov

$\mathrm{H}$

Katz

$=$ Hermannstadt $/$ Sibiu

$=$ Hermannstadt $/$ Sibiu

K1-Schenk

$=$ Katzendorf/Cața

$=$ Kronstadt $/$ Brașov

$\mathrm{Kr}$

$=$ Kleinschenk $/$ Cincşor $=$ Kronstadt $/$ Braşov

Lechn

= Kronstadt $/$ Braşov

$=$ Kronstadt $/$ Brașov

$=$ Lechnitz/Lechnița

= Bistritz/Nassod/Bistriţa/ Năsăud

$\mathrm{Lu}$

= Ludwigsdorf/Logig

= Mureș

Mard

= Mardisch/Moardăș

$=$ Hermannstadt/Sibiu

Med

$=$ Mediasch $/$ Mediaș

$=$ Hermannstadt/Sibiu

Mö

= Mönchsdorf/Herina

= Bistritz/Nassod/Bistriţa/

\section{Năsăud}

Msch

= Meschen/Moșna

$=$ Hermannstadt $/$ Sibiu

Nd-Ei

$=$ Niedereidisch/Ideciu de Jos = Mureș

Nd-Wall

$=$ Niederwallendorf/Vorstadt $\mathrm{v}$. Bistritz $=$ suburbie

a Bistriţei

Pas

$=$ Paßbusch/Posmuș

= Bistritz/Nassod/Bistriţa/ Năsăud

R-au

= Rosenau/Râşnov

= Kronstadt/Brașov

Reen

$=$ Reen/Reghin

= Mureș 


\begin{tabular}{|c|c|c|}
\hline Rosch & = Rosch/Răvășel & $=$ Hermannstadt $/$ Sibiu \\
\hline Schbg & $=$ Schäßburg/Sighișoara & $=$ Mureș \\
\hline Schbk & $=$ Schönbirk/Sigmar & $\begin{array}{l}=\text { Bistritz/Nassod/Bistriţa/ } \\
\text { Năsăud }\end{array}$ \\
\hline S-Gg & $\begin{aligned}= & \text { Sankt Georgen/ } \\
& \text { SângeorzuNou }\end{aligned}$ & $\begin{aligned}= & \text { Bistritz/Nassod/Bistriţa/ } \\
& \text { Năsăud }\end{aligned}$ \\
\hline Tra & $=$ Trappold $/$ Apold & $=$ Mureș \\
\hline Tschi & $=$ Tschippendorf/Cepari & $\begin{aligned}= & \text { Bistritz/Nassod/Bistriţa/ } \\
& \text { Năsăud }\end{aligned}$ \\
\hline Wall & $=$ Wallendorf/Unirea & $\begin{aligned}= & \text { Bistritz/Nassod/Bistriţa/ } \\
& \text { Năsăud }\end{aligned}$ \\
\hline Wbch & $=$ Weidenbach/Ghimbav & $v=$ Kronstadt $/$ Brașov \\
\hline Win & $=$ Windau/Ghinda & $\begin{aligned}= & \text { Bistritz/Nassod/Bistriţa/ } \\
& \text { Năsăud }\end{aligned}$ \\
\hline W1 & $=$ Weilau/Uila & $=$ Mureş \\
\hline Wm & $=$ Wermesch/Vermeș & $\begin{aligned}= & \text { Bistritz/Nassod/Bistriţa/ } \\
& \text { Năsăud }\end{aligned}$ \\
\hline Zen & $=$ Zendersch/Senereuş & $=$ Mureș \\
\hline Zep & $=$ Zepling/Dedrad & $=$ Mureș \\
\hline
\end{tabular}

\section{Literatur}

\section{Primärliteratur}

Derzsi, Julia (Hg.): Quellen zur Geschichte der Stadt Kronstadt Band X-Das Gerichtsbuch des Kronstädter Rates (15581580). Kronstadt 2016.

Haldenwang, Sigrid: Das Siebenbürgisch-Sächsische in der Sprachlandschaft Siebenbürgen. In: Kriegleder, Wynfrid et al. (Hgg.): Deutsche Sprache und Kultur - Presse-Literatur - Geschichte in Siebenbürgen. Bremen 2009, S. 11-23. Hillner, Johann: Volksthümlicher Glaube und Brauch bei Geburt und Taufe im Siebenbürger Sachsenlande. In: Programm des evang. Gymansiums in Schässburg und der damit verbundenen Lehranstalten (Schuljahr 1876/77), S. 46. 
Honterus, Johannes: Ausgewählte Schriften. Hrsg. von Oskar Netoliczka. Hermannstadt 1898.

Keintzel, Georg: Nösner Idiotismen. In: Festgabe der Stadt Bistritz. Bistritz 1897, S. 48.

Kisch, Gustav: Nösner Wörter und Wendungen. Ein Beitrag zum siebenbürgisch-sächsischen Wörterbuch. Bistritz 1900. Korrespondenzblatt des Vereins für siebenbürgische Landeskunde 1902.

Nationalarchiv Hermannstadt (früher Archiv der Stadt Hermannstadt und der Nationsuniversität).

Nordsiebenbürgisch-Sächsisches Wörterbuch.Bd. 1 (A-C) von Krauß, Friedrich, bearb. v. Richter, Gisela Köln 1986, Bd. 2 (D-F) Köln 1990, Bd. 3 (H-M) Köln 1993, Bd. 4 (N-Sch) Köln 1995, Bd. 5 (Se-Z) Köln 2006. Bd. 2-4 bearb. v. Richter, Gisela aufgrund der nachgelassenen Sammlungen von Krauß, Fr.; Bd. 5 bearb. v. Richter, G. †

$u$. Feßler, Helga aufgrund der nachgelassenen Sammlungen v. Krauß, Fr. [NSSWB].

Rechnungen aus dem Archiv der Stadt Kronstadt. Bd. 3 (15111571). Kronstadt 1896.

Siebenbürgische Vierteljahresschrift 1931.

Siebenbürgisch-Sächsisches Wörterbuch. Bisher 10 Bde erschienen. Bd. 1 (A-C) bearb. v. Schullerus, Adolf, Bd. 2 (D-F) bearb. v. Schullerus, A., Hofstädter, Friedrich u. Keintzel, Georg. Berlin 1924, 1926 (in einzelnen Lieferungen schon ab 1908 erschienen); Bd. 5 [R-Salarist: alte Zählung] bearb. v. Roth, Johann, Göckler, Gustav, Berlin 1929-1931. Weitergeführt v. einem Wörterbuchteam: Bd. 3 (G), Bd. 4 (H-J), Bd. 5 [K: neue Zählung] Berlin 19711975; Bd. 6 (L) 1993, Bd. 7 (M) 1998, Bd. 8 (N-P) 2002, Bd. 9 (Q-R) 2006, Bd. 10 (S-Sche) Bukarest 2014 [wird fortgesetzt]. [SSWB]. 


\section{Sekundärliteratur}

Benkö, Loránd et al. (Hgg.): A magyar nyelv történeti-etimológiai szótára (Historisch- etymologisches Wörterbuch der ungarischen Sprache). 3 Bde. Budapest 1970.

Dargiewicz, Anna: Fremde Elemente in Wortbildungen des Deutschen. $\mathrm{Zu}$ Hybridbildungen in der deutschen Gegenwartssprache am Beispiel einer raumgebundenen Untersuchung in der Universitäts- und Hansestadt Greifswald. In: Grabarek, Józef (Hg.): Schriften zur diachronen und synchronen Linguistik. Bd. 10. Frankfurt am Main 2013, S. 93. Fleischer, Wolfgang: Wortbildung der deutschen Gegenwartssprache. Leipzig 1974.

Fleischer, Wolfgang/Barz, Irmhild: Wortbildung der deutschen Gegenwartssprache. Tübingen 1992.

Halász, Előd: Magyar-Német Szótár (Ungarisch-Deutsches Wörterbuch). 2 Bde. Budapest ${ }^{11} 1994$.

Kluge-Etymologisches Wörterbuch der deutschen Sprache.

Bearb. v. Elmar Seebold. Berlin ${ }^{23} 1995$.

Rheinisches Wörterbuch, bearb u. hrsg. v. Josef Müller. Bd. 2 (E-G). Berlin 1931.

Schmeller, J(ohann) Andreas: Bayerisches Wörterbuch. Bd. 1 bearb. v. Karl Fromann. München 1872.

Tiktin, Hariton: Rumänisch-Deutsches Wörterbuch. Bd. 1 (A-

C). Wiesbaden ${ }^{2} 1986$.

Wahrig, Gerhard et al. (Hgg.): Brockhaus Wahrig-Deutsches

Wörterbuch, Bd. 3 (G-JZ). Wiesbaden 1981. 